\title{
Commentaries
}

\section{Counting the cost of proton pump inhibitors}

Proton pump inhibitors (PPIs) have become an indispensable part of the armoury of treating gastro-oesophageal reflux disease (GORD). They are more effective than $\mathrm{H}_{2}$ receptor antagonists and prokinetic agents in oesophagitis and endoscopy negative reflux disease. ${ }^{1}$ This efficacy comes at a price however and PPIs are the most expensive class of drug in the UK, costing nearly $£ 300$ million in 1998. ${ }^{2}$ There have been attempts to curb PPI expenditure and the UK National Institute for Clinical Excellence has issued guidelines on appropriate prescription. ${ }^{2}$

The problem is that there is a dearth of health economic data to inform those making health care decisions. Economic models have suggested that PPIs are cost effective therapy for GORD but there have been criticisms of this approach ${ }^{3}$ and they do not address the root of the problem. Economic decisions are simple when a drug that is more expensive and less effective is compared with a cheaper more effective agent. The latter is said to "dominate" the former and it requires little health economic expertise to realise that the cheaper drug should be used. Problems arise when a more expensive therapy is more effective than the cheaper option. The choice is less obvious in these circumstances and the decision depends on how much patients would be willing to pay to cure their symptoms. If they are willing to pay more than the extra cost per extra cure conferred by the more expensive therapy then it should be instituted, otherwise the cheaper drug should be prescribed. The choice of therapy is likely to depend on the severity of symptoms and once these interfere sufficiently with quality of life PPIs will represent value for money. There have been approximately 5000 publications evaluating PPIs and yet none has assessed how severe GORD symptoms need to be before patients are willing to pay for these drugs compared with cheaper alternatives.

The imbalance between the clinical and health economic information relating to PPIs has been partially redressed by Myrvold et al in this issue of Gut (see page 488). ${ }^{4}$ They have performed a rigorous randomised controlled trial comparing omeprazole with open antireflux surgery. There was no statistically significant difference in relapse rates between the PPI and surgery arm so the two interventions were considered to have a similar efficacy. If both treatments work equally well, the amount patients are willing to pay for cure of their symptoms becomes less relevant and the cheapest strategy should be the most efficient. This type of economic evaluation is termed a cost minimisation analysis. Myrvold et al followed patients for five years and found that direct medical costs were statistically significantly higher in the surgery than the medical arm in Sweden, Denmark, and Norway but not in Finland. These data suggest long term PPI therapy is more cost effective than antireflux surgery in most countries studied.

These results should however be interpreted cautiously. The main concern is that a cost minimisation analysis was performed. This is rarely an appropriate form of economic analysis ${ }^{5}$ and in this study the absence of a statistically significant difference between the two interventions does not imply they have the same efficacy. Antireflux surgery may be more effective than PPI therapy but the trial did not have the power to detect the size of the effect. Indeed, heartburn scores were statistically significantly lower in the surgery arm compared with the omeprazole arm and the lack of difference between the two groups could be due to the artificial method the authors used to define treatment failure. ${ }^{6}$ An analysis that looked at costs and benefits with a confidence interval around the incremental cost effectiveness estimate would be the most appropriate method of presenting the data.

There are other concerns about the analysis of the data. Parametric tests were used to compare the costs between the two interventions. Cost data are usually highly positively skewed and parametric statistics are often inappropriate. ${ }^{8}$ There was also a moderate drop out rate in the treatment groups and these patients were assumed to have incurred no costs. There are accepted methods of dealing with incomplete follow up in economic analyses ${ }^{9}$ and it would be unusual to assume that these patients cost nothing.

There is also debate about the appropriateness of conducting an economic analysis alongside a randomised controlled trial evaluating the clinical efficacy of different treatments. ${ }^{10}$ The power of the trial is usually centred on the detection of differences in clinical outcomes and the sample size is rarely adequate for detecting differences in cost benefit. ${ }^{5}$ Economic trials need to be as pragmatic as possible while assessment of clinical outcome usually requires many more follow up visits and investigations than would occur in clinical practice. ${ }^{11}$ Myrvold et al have tried to control for this by not including protocol driven visits in their cost calculations. These extra visits may change patients' attitudes to their symptoms however and may also alter clinician management decisions. This may have an influence on the costs patients incur and the economic analysis may not reflect clinical practice.

Myrvold et al report one of the few economic analyses of PPI therapy using randomised controlled trial data. Economic evaluation of health care interventions present new challenges in trial design and analysis and this is a rapidly evolving discipline. ${ }^{7}$ The results are difficult to interpret but the authors should still be congratulated for providing data in this important field. Rationing is inevitable in health care and until we have more studies addressing the cost effectiveness of PPI therapy in GORD there will remain a tension between clinicians wanting to give the best drug to their patients and the health care payers wanting to cut costs.

P MOAYYEDI

General Infirmary at Leeds,

Great George Street, Leeds LS1 3EX, UK

paulmo@ulth.northy.nhs.uk

1 Katzka D, Delaney B, Forman D, et al. Gastro-oesophageal reflux disease. Clin Evidence 2000;4:253-65.

2 Guidance on the use of proton pump inhibitors in the treatment of dyspepsia. Technology Appraisal Guidance No 7. National Institute for Clinical Technology Appraisal Guidance No 7. National Institute for
Excellence, July 2000 (http://www.nice.org.uk/pdf/Proton.pdf).

3 Hillman AL, Eisenberg JM, Pauly MV, et al. Avoiding bias in the conduct Hillman AL, Eisenberg JM, Pauly MV, et al. Avoiding bias in the conduct
and reporting of cost-effectiveness research sponsored by pharmaceutical companies. N Engl f Med 1994;324:1362-5. 
4 Myrvold HE, Lundell L, Miettinen P, et al. The cost of long term therapy for gastro-oesophageal reflux disease: a randomised trial comparing omeprazole and open antireflux surgery. Gut 2001;49:488-95.

5 Drummond MF, Richardson WS, O'Brien BJ, et al. How to use an article on economic analysis of clinical practice. FAMA 1997;277:1552-7.

6 Lundell L, Miettinen P, Myrvold HE, et al. Long term management of gastro-oesophageal reflux disease with omeprazole or open antireflux surgery. Eur $\mathcal{F}$ Gastroenterol Hepatol 2000;12:879-87.

7 Briggs AH, Gray AM. Handling uncertainty in economic evaluations of healthcare interventions. BMF 1999;319:635-8.
8 Thompson SG, Barber JA. How should cost data in pragmatic randomised trials be analysed? BMF 2000;320:1197-200

Lin DY, Feuer EJ, Etzioni R, et al. Estimating medical costs from incomplete follow-up data. Biometrics 1997;53:419-34

10 Coyle D, Davies L, Drummond MF. Emerging issues in designing economic evaluations alongside clinical trials. Int $\mathcal{f}$ Technol Assess Health Care 1998;14:135-44.

11 Drummond MF, Davies L. Economic analysis alongside clinical trials. Revisting the methodological issues. Int $\mathcal{F}$ Technol Assess Health Care 1991; 7:561-73.

\section{The external world of gluten and autoimmunity}

This commentary is not about autoimmune diseases, and therefore I will not discuss why a significant proportion of individuals are prone to develop autoimmune diseases. Suffice to say that this failure to spare self might represent an advantage when fighting infections. In autoimmunity there is a clear genetic involvement with a strong association between these diseases and some HLA alleles, ${ }^{1}$ but autoimmunity is not simply genetically controlled and environmental factors are essential. For example, in the syngenic NOD mice, the preferred animal model for type 1 diabetes, simple modifications in the "cleanness" of the housing conditions dramatically changes the incidence of the disease. ${ }^{2}$ More poignantly, in the context of a clinical setting, the concordance rate of autoimmune diseases in monozygous (genetically identical) twins is less than $50 \%{ }^{3}$ Hence environmental factor(s) are essential in induction of autoimmunity. Thus characterisation of "environmental" triggers and how they might promote autoimmunity is of paramount importance if we wish to understand, prevent, and eventually define strategies to treat these diseases. The gastrointestinal tract, with its vast surface of contact with the external world, represents the main door for the potential encounter of "environmental" triggers of autoimmunity.

To curb this risk, the gastrointestinal tract, as other mucosae, has devised means of inducing a protective response that however lacks the inflammatory flavour. In a simplistic way, antigens encountered on a mucosal surface trigger a Th2 (sometimes and depending on the authors Th3 or $\mathrm{T}$ regulatory) type of response instead of a proinflammatory Th1 response, dominant in autoimmune diseases. ${ }^{4}$ This mucosal characteristic has been exploited to protect or even treat autoimmune diseases via induction of mucosal tolerance. ${ }^{5}$

Some gastrointestinal diseases are associated with autoimmunity but coeliac disease (CD) has two important characteristics that make it a cut above the rest: the strongest HLA association and a single well defined triggergluten. ${ }^{6} \mathrm{CD}$ itself is a "spurious" autoimmune disease as it induces a reaction against self (antibodies against tissue transglutaminase) but this self aggression resolves on gluten withdrawal and hence strictly speaking does not qualify as an autoimmune disease.

Because we can control the encounter with the environmental factor (gluten) in CD, this is an ideal condition to study the relevance of the environment in induction of autoimmunity. Several years ago Ventura and colleagues postulated that the longer a coeliac patient encounters gluten, the greater is the chance that they will develop an autoimmune disease. ${ }^{7}$ In that report duration of gluten exposure was measured in children from birth to the time of diagnosis when gluten is normally removed from the diet. This line of reasoning is not too difficult to comprehend as it straightforwardly states that the longer is the exposure to the "toxic" agent the greater the potential cumulative effect. The implications however are enormous as we would have defined a single environmental factor and more importantly we could visualise how this promotes autoimmunity. For instance, we could envisage that in $\mathrm{CD}$ a rescheduling of the mucosal microenvironment is induced with a switch from the customarily "antiinflammatory" Th2 type milieu to a Th1 type, as observed in $\mathrm{CD} .{ }^{8}$ In the long run, this could tip the balance towards an autoimmune response although other conceivable explanations, such as increased mucosal permeability, are possible. In this issue of Gut, this important topic, which trespasses the boundaries of CD, has been revised (see page 502). ${ }^{9}$ Sategna Guidetti et al studied a group of adult coeliacs, rather than children, following a similar retrospective analysis implemented by Ventura and colleagues. ${ }^{7}$ The conclusions at first glance seem to be similar in the two reports: the later CD is diagnosed the greater the chance of a concomitant autoimmune disease. Sategna Guidetti et al however introduced a small "artifice" to reverse this apparently uncomplicated conclusion. Indeed, they argue that in a large group of coeliacs, diagnosis of the autoimmune disease preceded that of CD, and consequentially we should introduce this variable, among others, in analysis of the data. Surely if a patient becomes diabetic for example, 10 years before a diagnosis of CD, he/she did not require that extra time (10 years) of gluten challenge to develop diabetes. The authors thus reanalysed their data and surprisingly concluded that "actual gluten exposure", a concept that at a superficial reading of the paper is not easy to grasp, was not different between coeliacs with or without other autoimmune diseases. Thus they challenge the main conclusion of Ventura's paper. In evaluating both of these papers we need to consider several points which may help reconcile the different conclusions. For example, the two studies did not compare the same populations of coeliac patients. One study was conducted in children and the other in adults, and to date there is no consensus that child and adult CD are the same condition. That age might have an unforeseen effect in the progression of an immune mediated disease is again proved by the study of monozygotic twins discordant for type 1 diabetes. In that study it was demonstrated that the concordance rate dramatically decreased if age at diagnosis of the diabetic proband twin was made after the age of $24 .^{3}$ We also do not know whether, even in Ventura's study, the introduction of the concept "actual gluten exposure", at least for the groups of children diagnosed later in life, would have changed interpretation of the data. Clearly, the 
only experiment that can answer this question is not a retrospective but rather a prospective study, maintaining one portion of the young early diagnosed children on a gluten containing diet, but this is obviously impossible. We also do not know whether patients that have been diagnosed with CD at 36 years of age have had CD all their life, as there is evidence suggesting that other concomitant factors may start the clinical manifestations of CD.

As is often the case, this paper raises more questions than answers. One that is immediately obvious is why, regardless of the duration of "actual gluten exposure", coeliac patients with other autoimmune diseases are diagnosed later in life, implying that they may have a clinically less evident disease, making their diagnosis more difficult. Another question that has to be answered is whether or not the groups (with or without autoimmune diseases) are homogenous and what other parameters differentiate them apart from age at diagnosis. Indeed, we have to clarify why these late diagnosed patients are more prone to develop autoimmunity and how they differ compared with early diagnosed patients if we want to "make sense" of this finding. Therefore, functional, epidemiological, as well as genetic studies are required to unravel this puzzling question to shed light on the nature of the link between $\mathrm{CD}$ and autoimmune diseases and possibly to autoimmunity itself.

M LONDEI

Kennedy Institute of Rheumatology Division,

Imperial College School of Medicine,

1 Aspenlea Road, London W6 8LH, UK

m.londei@ic.ac.uk

$1 \mathrm{McDevitt} \mathrm{HO}$. The role of MHC class II molecules in susceptibility and resistance to autoimmunity. Curr Opin Immunol 1998;10:677-81.

2 Atkinson MA, Leiter EH. The NOD mouse model of type 1 diabetes: as good as it gets? Nat Med 1999;5:601-4.

3 Redondo MJ, Yu L, Hawa M, et al. Heterogeneity of type I diabetes: analysis of monozygotic twins in Great Britain and the United States. Diabetologia 2001:44:354-62.

4 Liblau RS, Singer SM, McDevitt HO. Th1 and Th2 CD4+ T cells in the pathogenesis of organ-specific autoimmune diseases. Immunol Today 1995; pathogen

5 Garcia G, Weiner HL. Manipulation of Th responses by oral tolerance. Curr Top Microbiol Immunol 1999;238:123-45.

6 Sollid LM, Thorsby E. HLA susceptibility genes in celiac disease: genetic mapping and role in pathogenesis. Gastroenterology 1993;105:910-22

7 Ventura A, Magazzu G, Greco L. Duration of exposure to gluten and risk for autoimmune disorders in patients with celiac disease. SIGEP Study Group for Autoimmune Disorders in Celiac Disease. Gastroenterology 1999; 117:297-303.

8 Nilsen EM, Jahnsen FL, Lundin KE, et al. Gluten induces an intestinal cytokine response strongly dominated by interferon gamma in patients with celiac disease. Gastroenterology 1998;115:551-63.

9 Sategna Guidetti C, Solerio E, Scaglione N, et al. Duration of gluten exposure in adult coeliac disease does not correlate with the risk for autoimmune disorders. Gut 2001;49:502-5.

\title{
7th European Forum on Quality Improvement in Health Care
}

\author{
21-23 March 2002 \\ Edinburgh, Scotland
}

We are delighted to announce this forthcoming conference in Edinburgh. Authors are invited to submit papers (call for papers closes on Friday 5 October 2001) and delegate enquiries are welcome.

The themes of the Forum are:

- Leadership, culture change, and change management

- Achieving radical improvement by redesigning care

- Health policy for lasting improvement in health care systems

- Patient safety

- Measurement for improvement, learning, and accountability

- Partnership with patients

- Professional quality: the foundation for improvement

- Continuous improvement in education and training

- People and improvement.

Presented to you by the BMJ Publishing Group (London, UK) and Institute for Healthcare Improvement (Boston, USA). For more information contact: quality@bma.org.uk or look at the website www.quality.bmjpg.com. Tel: +44 (0)20 7383 6409; fax: +44 (0)20 73736869 . 